\title{
SCHRÖDINGER EQUATIONS: POINTWISE CONVERGENCE TO THE INITIAL DATA
}

\author{
LUIS VEGA
}

(Communicated by Richard R. Goldberg)

\begin{abstract}
Let $u(x, t)$ be the solution of the Schrödinger equation with initial data $f$ in the Sobolev space $H^{s}\left(\mathbf{R}^{n}\right)$ with $s>\frac{1}{2}$. The a.e. convergence of $u(x, t)$ to $f(x)$ follows from a weighted estimate of the maximal function $u^{*}(x, t)=\sup _{t>0}|u(x, t)|$.
\end{abstract}

0. Let $f$ be in the Schwartz space $S\left(\mathbf{R}^{n}\right)$ and for $x \in \mathbf{R}^{n}, t \in \mathbf{R}$ set

$$
u(x, t)=\int_{\mathbf{R}^{n}} f(\xi) e^{i|\xi|^{2} t} e^{i x \cdot \xi} d \xi .
$$

It is well known that $u$ is the solution of the Schrödinger equation with initial data $f$.

$$
\Delta u=i \frac{\partial}{\partial t} u, \quad t>0, \quad u(x, 0)=f(x) .
$$

For $s \in \mathbf{R}$ we denote by $H^{s}\left(\mathbf{R}^{n}\right)$ the Sobolev space

$$
H^{s}\left(\mathbf{R}^{n}\right)=\left\{f \in S^{\prime}\left(\mathbf{R}^{n}\right) \text { s.t. }\|f\|_{H^{s}\left(\mathbf{R}^{n}\right)}=\left(\int\left(1+|\xi|^{2}\right)^{s}|\hat{f}(\xi)|^{2}\right)^{1 / 2}<+\infty\right\} .
$$

We obtain the following result.

THEOREM 1. Let $f$ be in $H^{s}\left(\mathbf{R}^{n}\right)$ with $s>\frac{1}{2}$. Then

$$
\lim _{t \rightarrow 0} u(x, t)=f(x) \text { a.e. } x \text {. }
$$

This result is a consequence of the boundedness of the maximal operator $u^{*}(x)=$ $\sup _{0<|t|}|u(x, t)|$.

THEOREM 2. Let $f$ be in $H^{s}\left(\mathbf{R}^{n}\right)$ with $s>a / 2$ and $a>1$. Then

$$
\left(\int\left|u^{*}(x)\right|^{2} \frac{d x}{(1+|x|)^{a}}\right)^{1 / 2} \leq c\|f\|_{H^{s}\left(\mathbf{R}^{n}\right)} .
$$

Since $H^{r}(\mathbf{R})$ with $r>\frac{1}{2}$ is embedded in $L^{\infty}(\mathbf{R})$ by the classical Sobolev inequalities, Theorem 2 is an immediate consequence of

THEOREM 3. If $0 \leq \alpha$ and $a>1$, then

$$
\left(\int_{\mathbf{R}^{n}} \int_{-\infty}^{\infty}\left|\frac{\partial^{\alpha}}{\partial t^{\alpha}} u(x, t)\right|^{2} d t \frac{d x}{(1+|x|)^{a}}\right)^{1 / 2} \leq c\|f\|_{H^{2 \alpha-1+a / 2}\left(\mathbf{R}^{n}\right)} .
$$

Received by the editors December 4, 1986.

1980 Mathematics Subject ('lassification (1985 Revision). Primary 42A45; Secondary $42 B 25$. 
The above results are best known for $n \geq 3$. For $n=1, \mathrm{~L}$. Carleson [2] proved the a.e. convergence for $f \in H^{1 / 4}\left(\mathbf{R}^{n}\right)$ and constructed an example of an $f \in H^{1 / 8}(\mathbf{R})$ such that $u(x, t)$ does not converge to $f$ a.e.

Later, B. Dahlberg and C. Kenig [5] proved that the positive result of Carleson was sharp.

These questions and the boundedness of the maximal operator have also been studied by C. Kenig and A. Ruiz [6], A. Carbery [1] and M. Cowling [4]. They prove that if $f \in H^{n / 4}\left(\mathbf{R}^{n}\right)$ or $f \in H^{s}\left(\mathbf{R}^{n}\right)$ for $s>1$, then the maximal function is bounded.

We also give an alternative proof to the fact that there is no boundedness for the maximal operator, and then no convergence result, for $f \in H^{s}\left(\mathbf{R}^{n}\right)$ with $s<\frac{1}{4}$ $(\S 1)$.

In $\S 2$, we generalize the above theorems to multipliers of the type $e^{i|\xi|^{b} t}$. These results have been obtained at the same time and independently by P. Sjölin.

We should like to thank S. Córdoba for his help and encouragement.

1. Proof of Theorem 3. We shall make use of the following inequalitiy which is proved below.

LEMMA. Let $g$ be in $L^{2}\left(S^{n-1}\right)$. Then if $a>1$

$$
\left(\int_{\mathbf{R}^{n}}\left|\int_{S^{n-1}} g(\xi) e^{i x \cdot \xi} d \sigma(\xi)\right|^{2} \frac{d x}{(1+|x|)^{a}}\right)^{1 / 2} \leq c\left(\int_{S^{n-1}}|g|^{2} d \sigma\right)^{1 / 2} .
$$

With a simple change of variable we obtain the representation in polar coordinates,

$$
u(x, t)=\frac{1}{2} \int_{0}^{\infty} e^{i s t} s^{n-2 / 2} \int_{S^{n-1}} f\left(s^{1 / 2} \xi\right) e^{i s^{1 / 2} x \cdot \xi} d \sigma(\xi) d s,
$$

and then we use Plancherel's inequality in the $t$ variable to get

$$
\begin{aligned}
& \int_{\mathbf{R}^{n}} \int_{-\infty}^{\infty}\left|\frac{\partial^{\alpha}}{\partial t^{\alpha}} u(x, t)\right|^{2} d t \frac{d x}{(1+|x|)^{a}} \\
& \quad=\frac{1}{4} \int_{\mathbf{R}^{n}} \int_{0}^{\infty}\left|s^{\alpha+(n-2) / 2} \int_{S^{n-1}} \hat{f}\left(s^{1 / 2} \xi\right) e^{i s^{1 / 2} x \cdot \xi} d \sigma(\xi)\right|^{2} d s \frac{d x}{(1+|x|)^{a}} \\
& \quad \leq \frac{1}{4} \int_{0}^{\infty}(1+s)^{a / 2+n / 2+2 \alpha-2} \int_{\mathbf{R}^{n}}\left|\hat{f}\left(s^{1 / 2}\right) e^{i x \cdot \xi} d \sigma(\xi)\right|^{2} \frac{d x}{(1+|x|)^{a}} d s
\end{aligned}
$$

by the lemma

$$
\begin{aligned}
& \leq c \int_{0}^{\infty}(1+s)^{a / 2+n / 2+2 \alpha-2} \int_{S^{n-1}}\left|\hat{f}\left(s^{1 / 2} \xi\right)\right|^{2} d \sigma(\xi) d s \\
& \leq c\|f\|_{H^{2 \alpha-1+a / 2}\left(\mathbf{R}^{n}\right)}^{2} .
\end{aligned}
$$

For $n=1$ the proof is similar. In this case one proves

$$
\left\|\left(\int_{-\infty}^{\infty}\left|\frac{\partial^{\alpha}}{\partial t^{\alpha}} u(x, t)\right|^{2} d t\right)^{1 / 2}\right\|_{L^{\infty}(\mathbf{R})} \leq c\|f\|_{H^{2 \alpha-1}(\mathbf{R})} .
$$

PROOF OF THE LEMMA. We want to see that $L^{2}\left(S^{n-1}\right)$ is embedded in $H^{-s}\left(\mathbf{R}^{n}\right)$ with $s>\frac{1}{2}$. Though this fact is an immediate consequence of the trace 
theorem, we give an alternative proof. We shall do this in $S^{1}$. The generalization to higher dimensions is straightforward.

It is sufficient to prove the inequalitiy for simple functions having their supports contained in $\left\{x=e^{i \theta}, 0 \leq \theta \leq \pi / 4\right\}$. Set $\delta>0$ and consider for every

$$
\tilde{R}_{j}=\left\{x=e^{i \theta} \in S^{1}: j \frac{\pi}{4\left[\delta^{-1 / 2}\right]} \leq \theta \leq(j+1) \frac{\pi}{4\left[\delta^{-1 / 2}\right]}\right\}
$$

$j \in\left\{0, \ldots,\left[\delta^{-1 / 2}\right]\right\}$. Each $\tilde{R}_{j}$ can be covered by a rectangle $R_{j}$ of dimensions $c_{1} \delta \times c_{2} \delta^{1 / 2}$ in the normal and tangent directions to $S^{1}$ at the point $\left.e^{i\left(j \pi / 4 \mid \delta^{-1 / 2}\right.} \mid\right)$ where $c_{1}, c_{2}$ are convenient universal constants greater than 1 (see the figure).

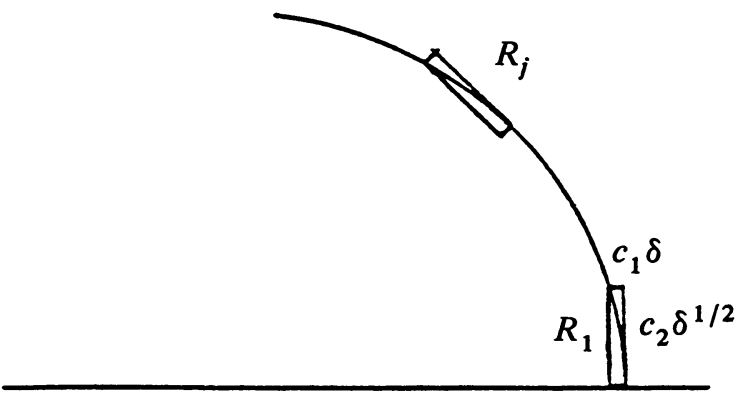

Then, it is sufficient to show

$$
\left(\int_{\mathbf{R}^{2}}\left|\frac{1}{\delta} \sum_{j} a_{j} \hat{\varphi}_{j}\right|^{2}(1+|x|)^{-a}\right)^{1 / 2} \leq c\left(\sum_{j}\left|a_{j}\right|^{2} \delta^{1 / 2}\right)^{1 / 2}
$$

where $\left\{\varphi_{j}\right\}$ is a smooth partition of unity subordinate to $\left\{R_{j}\right\}$ and $a_{j} \in \mathbf{C}$. But

$$
\begin{aligned}
\int_{\mathbf{R}^{2}}\left|\sum a_{j} \hat{\varphi}_{j}\right|^{2}(1+|x|)^{-a} & \leq \int\left|\sum_{j} a_{j} \hat{\varphi}_{j} \frac{1}{\left(1+\left|x_{1}\right|\right)^{a / 2}}\right|^{2} \\
& =\int\left|\sum_{j} a_{j} \varphi_{j} * \mu\right|^{2},
\end{aligned}
$$

where $\hat{\mu}(x)=1 /\left(1+\left|x_{1}\right|\right)^{a / 2}$. Then $\mu(\xi)$ is a measure which acts only on the $\xi_{1}$ direction and by the finite overlapping of the supports of $\varphi_{j}$,

$$
\begin{aligned}
\int\left|\sum_{j} a_{j} \varphi_{j} * \mu\right|^{2} & \leq 2 \sum_{j}\left|a_{j}\right|^{2} \int\left|\varphi_{j} * \mu\right|^{2} \\
& =2 \sum\left|a_{j}\right| \int\left|\hat{\varphi}_{j}\right|^{2} \frac{d x}{\left(1+\left|x_{1}\right|\right)^{a}} .
\end{aligned}
$$

Therefore, it is sufficient to see that

$$
\int\left|\hat{\varphi}_{j}\right|^{2} \frac{d x}{\left(1+\left|x_{1}\right|\right)^{a}} \leq c \delta^{5 / 2}
$$


If we call $\tau_{j}$ and $\eta_{j}$ the tangent and normal directions in $e^{i j \pi / 4\left[\delta^{-1 / 2}\right]}$ we can take $\varphi_{j}$ such that

$$
\left|\frac{\partial^{\alpha}}{\partial \tau_{j}^{\alpha}} \varphi_{j}(\xi)\right| \leq c \delta^{-\alpha / 2}, \quad\left|\frac{\partial^{\beta}}{\partial \eta_{j}^{\beta}} \varphi_{j}(\xi)\right| \leq c \delta^{-\beta} .
$$

Then, integrating by parts,

$$
\left|\hat{\varphi}_{j}(x)\right|=\left|\int \varphi_{j}(\xi) e^{-i x \cdot \xi}\right| \leq c 2^{-2 k} \delta^{3 / 2}
$$

for $k=0,1, \ldots$ and $x=x_{1} \tau_{j}+x_{2} \eta_{j} ; 2^{k} \delta^{-1} \leq x_{1} \leq 2^{k+1} \delta^{-1} ; 2^{k} \delta^{-1 / 2} \leq x_{2} \leq$ $2^{k+1} \delta^{-1 / 2}$. Since we always have $\left|\hat{\varphi}_{j}(x)\right|<c^{3 / 2}$, then

$$
\int\left|\hat{\varphi}_{j}\right|^{2}\left(1+\left|x_{1}\right|\right)^{-a / 2} \leq c \sum_{k} 2^{-3 k-1 / 2+3} .
$$

\section{Negative results.}

THEOREM 4. The inequality

$$
\left(\int_{B(0, R)}\left|u^{*}(x)\right|^{2} d x\right)^{1 / 2} \leq C_{R}\|f\|_{H^{s}\left(\mathbf{R}^{n}\right)}
$$

does not hold for $s<\frac{1}{4}$.

ProOF. Let $\phi_{k} \in C_{0}^{\infty}\left(\left[2^{k}, 2^{k}+2^{k / 2}\right]\right)$ with $k=0,1, \ldots$ and $0 \leq \phi_{k} \leq 1$, $\left|\left(d^{i} / d x^{i}\right) \phi_{k}(x)\right| \leq C 2^{-(k / 2) i}$. It is known that for $[x]$ sufficiently large

$$
\int_{S^{n-1}} e^{i x \cdot \xi} d \sigma(\xi)=\frac{1}{|x|^{(n-1) / 2}}\left\{c_{1} e^{i|x|}+c_{2} e^{-i|x|}\right\}+o\left(\frac{1}{|x|^{(n+1)}}\right) .
$$

Then

$$
\begin{aligned}
u_{k}(x, t)= & \int_{\mathbf{R}^{n}} e^{i|\xi|^{2} t} \phi_{k}(\xi) e^{i x \cdot \xi} d \sigma(\xi) \\
= & \frac{c}{|x|^{(n-1) / 2}} \int e^{i\left(r^{2} t-r|x|\right)} r^{(n-1) / 2} \phi_{k}(r) d r \\
& +\frac{c}{|x|^{(n-1) / 2}} e^{i\left(r^{2} t+r|x|\right)} r^{(n-1) / 2} \phi_{k}(r) d r \\
& +\frac{1}{|x|^{(n+1) / 2}} o\left(2^{k((n / 2)-1)}\right) \\
= & I_{1}(x)+I_{2}(x)+\frac{1}{|x|^{(n+1) / 2}} o\left(2^{k((n / 2)-1)}\right) .
\end{aligned}
$$

For every $x \in \mathbf{R}$ such that $\frac{1}{2} \leq|x| \leq 1$ we choose

$$
t_{x}=\frac{|x|}{2^{k+1}+2^{(k / 2)+1}} .
$$

Let us define $\eta_{1}(r)=r^{2} t_{x}-r|x|$. Then $\eta_{1}^{\prime}\left(2^{k}+2^{k / 2} / 2\right)=0$ and by the stationary phase lemma we can assure $I_{1}(x) \geq\left(c /|x|^{(n-1) / 2}\right) 2^{k(n / 2)}$.

Let $\eta_{2}(r)=r^{2} t_{x}+r|x|$. Then $\eta_{2}^{1}(r)>\frac{1}{2}$ and integrating by parts $\left|I_{2}(x)\right| \leq$ $C \cdot 2^{k((n / 2)-1)}$. Then $u_{k}^{*}(x) \geq C 2^{k(n / 2)}$ for $\frac{1}{2} \leq|x| \leq 1$. Since $\left\|\hat{\varphi}_{k}\right\|_{H^{*}\left(\mathbf{R}^{n}\right)} \leq$ $c 2^{k(s+n / 2-1 / 4)}$ we conclude that $s<\frac{1}{4}$ is necessary. 
FURTHER RESULTS. These theorems can be generalized to multipliers of the type $e^{i|\xi|^{b_{t}}}$. In the following we state the results we have obtained using the techniques presented above.

Let us define $u_{b}(x, t)=\hat{f}(\xi) e^{i|\xi|^{b} t} e^{i x \cdot \xi} d \sigma(\xi)$.

THEOREM 1'. For $s>\frac{1}{2}$ and $f \in H^{s}\left(\mathbf{R}^{n}\right)$

$$
\lim _{t \rightarrow 0} u_{b}(x, t)=f(x) \text { a.e. } x \text {. }
$$

THEOREM $2^{\prime}$. Let $u_{b}^{*}(x)=\sup _{t}\left|u_{b}(x, t)\right|$ and $a>1$. Then

$$
\left(\int_{\mathbf{R}^{n}}\left|u_{b}^{*}(x)\right|^{2} \frac{d x}{(1+|x|)^{a}}\right)^{1 / 2} \leq C\|f\|_{H^{s}\left(\mathbf{R}^{n}\right)} \quad \text { for } s>\frac{a}{2} .
$$

THEOREM $3^{\prime}$. For $a>1$ and $\alpha \geq 0$

$$
\left(\int_{\mathbf{R}^{n}} \int_{-\infty}^{\infty}\left|\frac{\partial^{\alpha}}{\partial t^{\alpha}} u_{b}(x, t)\right|^{2} d t \frac{d x}{(1+|x|)^{a}}\right)^{1 / 2} \leq C\|f\|_{H^{(\alpha-1 / 2) b+a / 2}\left(\mathbf{R}^{n}\right)} .
$$

THEOREM $4^{\prime}$. Let $b>1$. The inequality

$$
\left(\int_{B(0, R)}\left(\sup _{0<t<1}\left|u_{b}(x, t)\right|\right)^{2} d x\right)^{1 / 2} \leq C_{R}\|f\|_{H^{s}\left(\mathbf{R}^{n}\right)}
$$

does not hold for $s<\frac{1}{4}$.

\section{REFERENCES}

1. A. Carbery, Radial Fourier multipliers and associated maximal functions, Recent Progress in Fourier Analysis, North-Holland Mathematics Studies, North-Holland, Amsterdam, p. 111.

2. L. Carleson, Some analytical problems re'ated to statistical mechanics, Euclidean Harmonic Analysis, Lecture Notes in Math., vol. 779, Springer-Verlag, Berlin and New York, 1979, pp. 5-45.

3. A. Córdoba, A note on Bochner-Riesz operators, Duke Math. J. 46 (1979), 505-511.

4. M. Cowling, Pointwise behaviour of solutions to Schrödinger equations, Harmonic Analysis, Lecture Notes in Math., vol. 992, Springer-Verlag, Berlin and New York, 1983, pp. 83-90.

5. B. Dahlberg and C. Kenig, $A$ note on the almost everywhere behaviour of solutions to the Schrödinger equation, Harmonic Analysis, Lecture Notes in Math., vol. 908, Springer-Verlag, Berlin and New York, 1982, pp. 205-208.

6. C. Kenig and A. Ruiz, A strong type $(2,2)$ estimate for a maximal operator associated to the Schrödinger equation, Trans. Amer. Math. Soc. 280 (1983), 239-246.

7. P. Sjölin, Regularity of solutions to the Schrödinger equation, Duke Math. J. 55 (1987), 699-715.

División de Matemáticas, Universidad Autónoma, 28049 Madrid, SPAin 